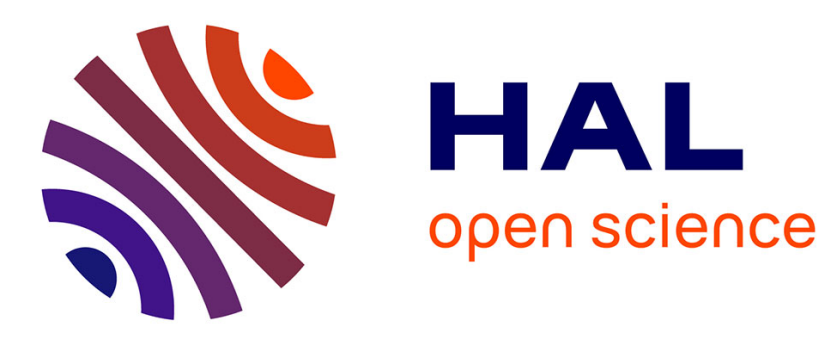

\title{
Spin-bottleneck due to spin-charge separation in a superconductor
}

\author{
Brigitte C. Leridon, J. Lesueur, M. Aprili
}

\section{To cite this version:}

Brigitte C. Leridon, J. Lesueur, M. Aprili. Spin-bottleneck due to spin-charge separation in a superconductor. Physical Review B: Condensed Matter and Materials Physics (1998-2015), 2005, 72 (18), pp.180505. 10.1103/PHYSREVB.72.180505 . hal-00910914

\section{HAL Id: hal-00910914 https://hal.science/hal-00910914}

Submitted on 9 Jul 2019

HAL is a multi-disciplinary open access archive for the deposit and dissemination of scientific research documents, whether they are published or not. The documents may come from teaching and research institutions in France or abroad, or from public or private research centers.
L'archive ouverte pluridisciplinaire HAL, est destinée au dépôt et à la diffusion de documents scientifiques de niveau recherche, publiés ou non, émanant des établissements d'enseignement et de recherche français ou étrangers, des laboratoires publics ou privés. 


\title{
Spin-bottleneck due to spin-charge separation in a superconductor
}

\author{
B. Leridon,* J. Lesueur, and M. Aprili \\ Laboratoire de Physique Quantique, UPR5, ESPCI/CNRS, 10 rue Vauquelin, 75005 Paris, France
}

(Received 21 October 2005; published 28 November 2005)

\begin{abstract}
An experimental device was designed to measure the effect of the injection of spin-polarized carriers on the superconductive gap and density of states (DOS). Quasiparticles were injected from a ferromagnet $\left(\mathrm{Ni}_{0.8} \mathrm{Fe}_{0.2}\right)$ through a tunnel junction into a conventional superconductor $(\mathrm{Nb})$, while charge neutrality was maintained by a supercurrent. The DOS of the superconductor was measured through a second tunnel junction with a normal paramagnetic metal. No significant decrease of the superconductive gap was observed while a noticeable heating of the quasiparticles of the superconductor was measured. A similar experiment performed with current injected from a paramagnet ( $\mathrm{Al}$ or $\mathrm{Ag}$ ) showed no heating of quasiparticles. These observations are consistent with spin-charge separation of Bogoliubov quasiparticles and spin-bottleneck due to the enhanced recombination time of pure spin excitations.
\end{abstract}

DOI: 10.1103/PhysRevB.72.180505

PACS number(s): 74.50. $+\mathrm{r}, 74.40 .+\mathrm{k}, 74.78 . \mathrm{Na}$

We have designed an experiment to probe the density of states of a superconductor when current is injected from a ferromagnet through a tunnel junction. When an electron is injected at the gap energy into a superconductor, its spin goes to the corresponding excitation, while its charge goes entirely to the condensate. Therefore at the gap edge a Bogoliubov quasiparticle carries only spin and no charge. This point has been reemphasized by Refs. 1 and 2 . At high energies compared to the gap, however, a quasiparticle in a superconductor is similar to a metallic excitation and carries both spin and charge. At such energies, an entering electron transfers no charge to the condensate but as the quasiparticle relaxes towards the energy gap, its charge is transferred to the superfluid without change in spin. The properties of the superfluid enable the charge to be evacuated from near the injection region without any resistance. The spin excitations at the gap edge are therefore accumulated in the superconductor near the injection junction and spatially decoupled from the charge, provided their recombination rate be sufficiently large. In the present experiment, these pure-spin excitations are also spin polarized. This spin polarization relaxes over a time $\tau_{s r}$, equal to infinity if no magnetic impurities and no spin orbit are present. In reality, if $\tau_{s r}$ is only larger than the recombination time $\tau_{r}$, then the recombination process is controlled by $\tau_{s r}$ and the existence of pure-spin excitations can be favored. The present paper realizes this experimental situation. Spin-polarized quasiparticles are injected from a ferromagnet $\left(\mathrm{Ni}_{0.8} \mathrm{Fe}_{0.2}\right)$ into the superconductor $(\mathrm{Nb})$. The charge is evacuated through the superconducting condensate by the supercurrent (over the penetration depth $50 \mathrm{~nm}$ ) while the spin excitations diffuse within a limited volume in which a second tunnel junction acts as an independent detector. The experiment differs from the pioneer experiment performed by Johnson ${ }^{3}$ who estimated the spin relaxation length to be about $1 \mu \mathrm{m}$ in $\mathrm{Nb}$, because in the present paper, Andreev reflection processes are suppressed by the use of a tunnel junction at the injection. It also differs from the experiments done by Meservey and Tedrow as no Zeemann splitting of the density of states (DOS) is considered here. 4
Experimental setup. Figure 1(a) shows a schematic side view of the device while Fig. 1(b) is a top view. All layers are $50 \mathrm{~nm}$ thick and were deposited in situ using electron gun evaporation at about $10^{-8}$ Torr on a Si substrate. Different patterns were defined for the metal and oxide layers by use of mechanical masks.

The "detector" junction at the bottom of the device is an $\mathrm{Al} / \mathrm{Al}_{2} \mathrm{O}_{3} / \mathrm{Nb}$ junction with an area of about $750 \mu \mathrm{m} \times 750 \mu \mathrm{m}$ and resistance of about $100 \Omega$. The superconducting layer consists of a 50-nm-thick $\mathrm{Nb}$ layer $\left(T_{C}=8.2 \mathrm{~K}\right)$, on top of which is deposited a 50 -nm-thick Al layer, which is experimentally shown to be superconducting by proximity to the $\mathrm{Nb}$. The top "detector" junction is again $750 \mu \mathrm{m} \times 750 \mu \mathrm{m}$ wide but its resistance at high energy is only about $1 \Omega$. The upper layer is either a ferromagnet $\left(\mathrm{Ni}_{0.8} \mathrm{Fe}_{0.2}\right)$ that was magnetized by applying a $6000-\mathrm{G}$ inplane magnetic field, or a paramagnet ( $\mathrm{Al} \mathrm{or} \mathrm{Ag}$ ) for comparison.

The ac conductance of the detector was measured as a function of the dc bias voltage at about $1.5 \mathrm{~K}$ in a pumped

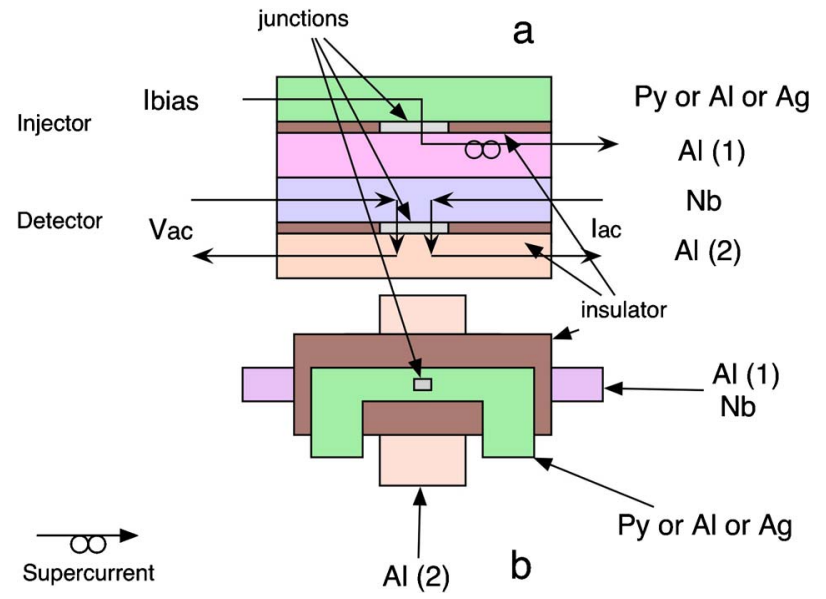

FIG. 1. (Color online) Sketch of the device. a) Side view (substrate is omitted). b) Top view (on a different scale). All layers are $50 \mathrm{~nm}$ thick. Junctions are $750 \mu \mathrm{m}$ wide. Py denotes the permalloy $\mathrm{Ni}_{0.8} \mathrm{Fe}_{0.2}$. 


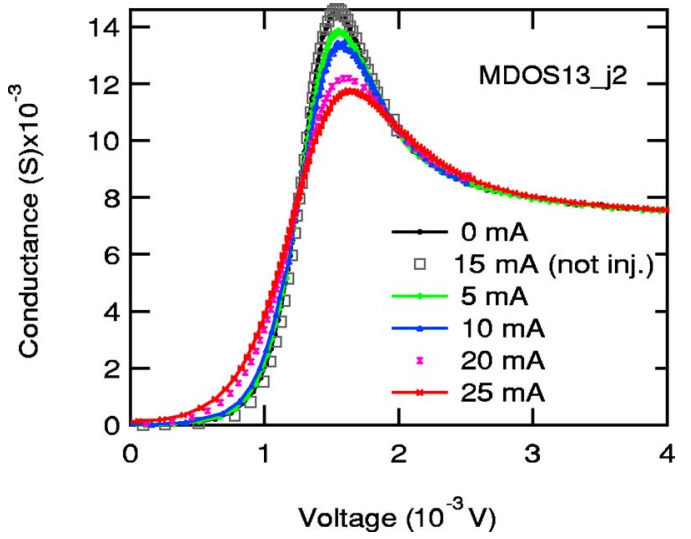

FIG. 2. (Color online) $d I / d V$ curves of the detector in the case of ferromagnet injection, for different values of the bias current through the injector. $T_{b a t h}=1.48 \mathrm{~K}$. The spectrum is displayed only for positive voltages but was symmetric for negative voltages. The open gray squares correspond to a situation where the current flows through the ferromagnet layer but not through the junction, allowing to rule out spurious Joule effects through the circuitry.

helium bath dewar, for different values of the injection current ranging from $-25 \mathrm{~mA}$ to $+25 \mathrm{~mA}$. In the case when the injection is made through a ferromagnet injector, the spectra appear to be strongly modified (see Fig. 2) in the same way for both directions of the injected current. However, when the current is applied through the paramagnet injector into the superconductor, no effect is seen on the $d I / d V$ curves of the detector (see Fig. 3).

Figure 4 illustrates a typical comparison for a ferromagnet injection with respect to a paramagnet injection, where the conductance at zero energy is being mesured as a function of the bias current. Whereas in Fig. 4 no effect is visible for the paramagnet injection, actually a tiny effect of increase of the zero voltage conductance (and correlated decrease of the gap voltage conductance) was measured with a magnitude of $1 \%$ of variation at $25 \mathrm{~mA}$, i.e., 400 times smaller than in the ferromagnet case.

These tests were made on several different devices with either ferromagnet or paramagnet injectors and different val-

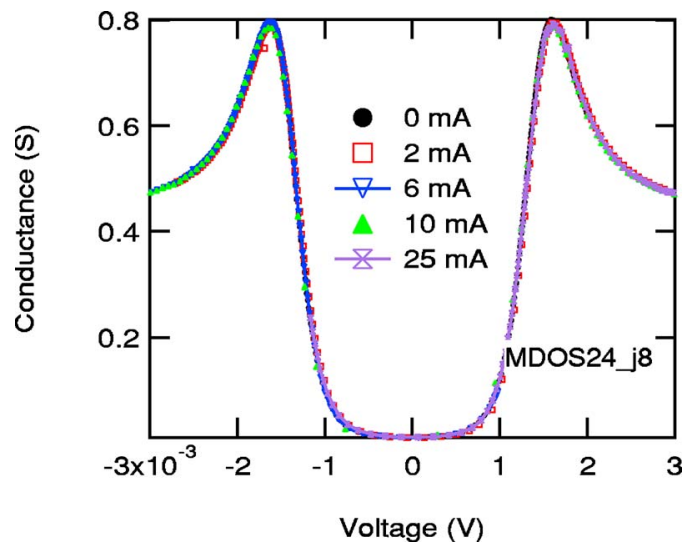

FIG. 3. (Color online) $d I / d V$ curves of the detector in the case of paramagnet injection, for different values of the injection current. All curves are superimposed. $T_{\text {bath }}=1.7 \mathrm{~K}$.

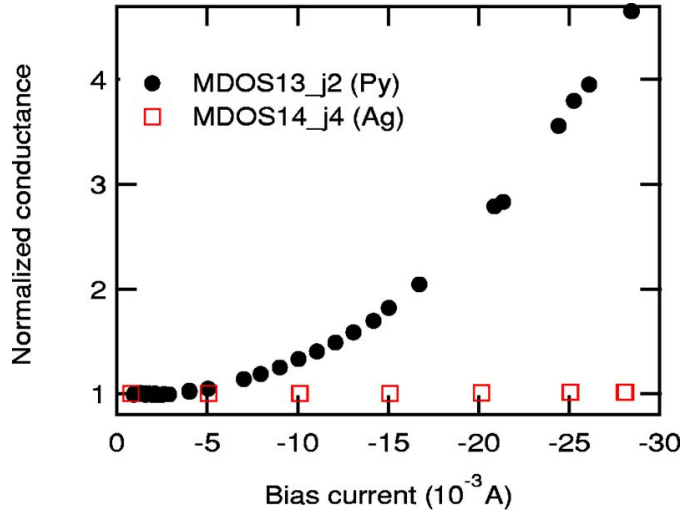

FIG. 4. (Color online) Variation of $d I / d V$ at zero voltage of the detector for a ferromagnet injector (black dots) and a paramagnet injector (open red squares). The conductance was normalized to its zero bias current value. The resistances of the injector junctions are, respectively, 2.6 and $1.5 \Omega$.

ues of the injector tunneling resistance. The existence or absence of the effect was correlated only to the nature of the top electrode. In order to rule out spurious heating effect, such as phonons generated in the top junction by Joule effect, a test experiment was carried. The sample with a ferromagnet injector was cooled down slightly below the critical temperature of the $\mathrm{Al}$ electrode of the detector (to $1.3 \mathrm{~K}$ ). A current was applied through the injector junction but instead of measuring the $d I / d V$ curve of the detector we rather measured the resistance of the $\mathrm{Al}$ strip. Any local increase of the temperature of more than $50 \mathrm{mK}$ due to a heating of the $\mathrm{Al}$ strip should result in nonzero resistance. No such effect was observed for injection currents up to $20 \mathrm{~mA}$. This implies that there was a gradient in the electronic temperature of the $\mathrm{Al}$ of about $1 \mathrm{~K}$ over $1 \mathrm{~mm}$. If the mechanism at work was phonons generated in the the top junction that consequently excite electrons, then there would be a gradient of equal magnitude also in the phonon temperature. Due to the high thermal conductivity of the Si wafer on which the Al strip is deposited, this would require a value of the power delivered to the top junction of $10^{-1} \mathrm{~W}$ so $10^{3}$ larger than the actual value. Therefore it is only a gradient in the electronic temperature and the mechanism has to be a direct excitation of quasiparticles that may eventually excite some phonons. The power delivered to the quasiparticles in the injector must roughly equal the power needed to ensure the gradient of electronic temperature. This is met for an estimated value of the electronic thermal conductance of the Al strip of $10^{-5} \mathrm{~W} \mathrm{~cm} \mathrm{~K}^{-1}$ corresponding to a thermal conductivity of $10 \mathrm{~W} \mathrm{~cm}^{-1} \mathrm{~K}^{-1}$.

Data analysis. The principal observation is the considerable difference between the spectra obtained on the junctions where the injector is a ferromagnet or a paramagnet. The spectra are shown to be strongly dependent on the injected spin-polarized current. Actually they are found to be very well fitted by a conventional BCS tunneling expression with a temperature higher than the measured He-bath temperature (see an example in Fig. 5 and the fitting parameters in Table I). One may recall here that an equilibrium BCS junction constitutes by itself an idealized thermometer for the elec- 


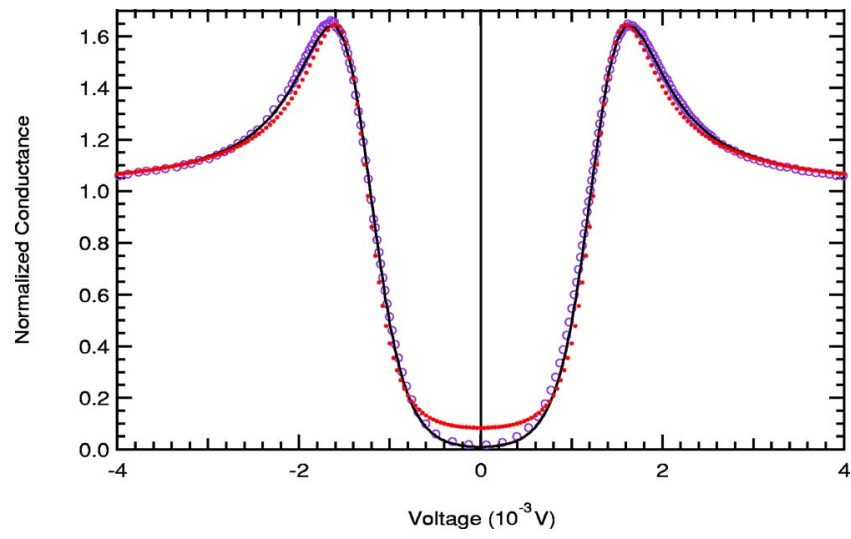

FIG. 5. (Color online) Fit using BCS theory of the spectrum measured under a spin-polarized current of $25 \mathrm{~mA}$. Blue circles: experimental data; line, BCS fit using $T^{*}=2.38 \mathrm{~K}$ and $\Delta=1.37 \mathrm{meV}$; red squares, attempt using Dynes model $^{6}$ with $\Gamma=110 \mu \mathrm{eV}, T^{*}=T=1.48 \mathrm{~K}$, and $\Delta=1.38 \mathrm{meV}$.

trons. Once the conductance is normalized to its value at high temperature and the gap to its zero-temperature value, the curve depends only on the temperature of the metal. It is quite remarkable that such a simple result is being obtained. The gap amplitude shows only a slight decrease (See Table I). This indicates that injected itinerant electrons do behave quite differently from localized magnetic moments. The expression giving the conductance through a tunnel junction in the framework of BCS theory is the following, ${ }^{5}$ but please see Ref. 6

$$
G(V)=-\frac{4 \pi e^{2}}{\hbar}|T|^{2} D_{N}\left(E_{F}\right) \int_{0}^{\infty} D_{S}(E) \frac{\partial f}{\partial E}(E+e V) d E
$$

where $D_{S}(E)$ is the density of states of the superconductor, $D_{N}(E)$ of the normal metal, and $f(E)$ refers to the FermiDirac distribution on the metal side.

Interpretation. The net effect of injecting a spin-polarized current is therefore observed to be an enhanced electronic temperature of the metal.

It has been observed in previous experiments ${ }^{7,8}$ that any massive injection of quasiparticles in a superconductor leads to an out-of-equilibrium state where excitations stay at a high

TABLE I. Results of the fits to a BCS tunneling conductance curve.

\begin{tabular}{cccc}
\hline \hline $\begin{array}{c}\text { Bias current } \\
(\mathrm{mA})\end{array}$ & $\begin{array}{c}T_{\text {bath }} \\
(\mathrm{K})\end{array}$ & $\begin{array}{c}T^{*} \\
(\mathrm{~K})\end{array}$ & $\begin{array}{c}\Delta \\
(\mathrm{meV})\end{array}$ \\
\hline & $\pm 5 \mathrm{mK}$ & $\pm 80 \mathrm{mK}$ & $\pm 10 \mu \mathrm{eV}$ \\
0 & 4.21 & 4.21 & 1.28 \\
0 & 1.48 & 1.48 & 1.38 \\
4.98 & 1.47 & 1.61 & 1.38 \\
9.99 & 1.47 & 1.71 & 1.38 \\
14.72 & 1.47 & 1.75 & 1.375 \\
19.95 & 1.47 & 2.10 & 1.37 \\
25 & 1.48 & 2.38 & 1.37 \\
\hline \hline
\end{tabular}

temperature, thermalized with high-energy phonons (phonons with energies greater than $2 \Delta$ ), while the condensate remains in equilibrium with low energy phonons. This imbalance is controlled by the injection rate of quasiparticles (and to a certain extent by their energies when entering the superconductor) and by the recombination time of the quasiparticles $\tau_{r}$. The phonon escape time may also play a role and contribute to a greater effective $\tau_{r}$. Similar out-ofequibrium effects without spin polarization were very carefully studied experimentally by different groups ${ }^{9-13}$ and theoretically by Refs. 8, 14, and 15 .

In the case of spin-polarized injection, the actual $\tau_{r}$ is substantially modified for a majority of the excitations due to their spin polarization. Two quasiparticles with the same spin polarization cannot recombine to the condensate. Therefore, if the excitations of the superconductor have a polarization (fraction of majority spins) $P$, then the fraction $2(1-P)$ recombine over a time $\tau_{r}$, and the fraction $2 P-1$ must experience spin flip before recombining, i.e., will recombine over $\tau_{r}+\tau_{s r}$. If the spin relaxation time $\tau_{s r}$ is much greater than $\tau_{r}$ and the inelastic scattering time $\tau_{s}$, they come to equilibrium at a temperature $T^{*}$ and a chemical potential $\mu^{*}$ different from that of the condensate. The "bottleneck" is then the spin relaxation.

The most likely source of spin polarization relaxation in $\mathrm{Nb}$, is spin-orbit scattering from heavier impurities (like $\mathrm{Ta}$ ). This was measured many years ago ${ }^{16}$ in a limited temperature range near $T_{c}$ and a theory was provided by Yafet. ${ }^{17}$ The relaxation time diverges at low $T$ because of the BCS coherence factor, which takes into account that the matrix element for spin-orbit scattering renormalizes to zero at the gap edge because the excitations at such energy do not carry charge. Spin-charge separation therefore modifies the spin dynamics in the spin-charge (SC) state and induces a larger $\tau_{s r}$. $\tau_{s r}$ will vary from sample to sample but taking the measurements, ${ }^{16}$ and extrapolating using the theory by Yafet, we estimate $\tau_{s r}$ at $2 \mathrm{~K}$ in $\mathrm{Nb}$ to be about $10^{-8} \mathrm{~s}$. Johnson ${ }^{13}$ has measured $\tau_{r}$ in $\mathrm{Nb}$ down to $T / T_{c}=0.78$ and finds it at the lowest temperature to be about $10^{-11} \mathrm{~s}$. Using this value and extrapolating, following Kaplan et al. ${ }^{18}$ to a temperature of $2 \mathrm{~K}, \tau_{r}$ is estimated to be about $10^{-9} \mathrm{~s}$. It may actually be even smaller because what is calculated in Kaplan's work is the $\tau_{r}$ for two quasiparticles, the rest of the particles being at equilibrium. Seminozhenko ${ }^{19}$ has shown that in the case of an out-ofequilibrium distribution $\tau_{r}$ is further reduced.

$\tau_{s}$ has been calculated also for a quasiparticle at a given energy, the lattice being at equilibrium. ${ }^{18}$ For a particle injected at energy $2 \Delta, \tau_{s}$ is about $6 \times 10^{-11} \mathrm{~s}$. Of course it varies with the energy of the injected quasiparticle and has not been calculated for the present case of a high density of quasiparticles at a high effective temperature together with hot thermalized phonons. In our case, the thermalization may also be determined by particle-particle scattering. ${ }^{20}$ The fact that our tunneling curves fit a "thermal distribution" is an evidence that the thermalization time is substantially shorter than the spin flip blocked recombination time. Parker ${ }^{8}$ has previously observed the effects of an increased quasiparticle density in an experiment in which the bottleneck is the phonon escape time rather than the spin-flip time as in our case. We may estimate, using his procedure that the density of 
excess quasiparticles for an injection current of $20 \mathrm{~mA}$ is about 80 times the expected number at the bath temperature. The out-of-equibrium excitations adopt a thermal distribution characterized by a temperature $T^{*}$ and a chemical potential $\mu^{*}$. We may estimate the change $\mu^{*}-\mu$ to be of the order of the chemical potential shift observed in charge-imbalance experiments (less than $1 \mathrm{nV}$ ), consistent with its lack of importance in our experiment compared to that of $T^{*}$.

Our experiment shows through the fit to Eq. (1) shown in Fig. 5, that the detector electrode $\mathrm{Al}$ at least in the vicinity of the tunneling region also comes to thermal equilibrium with the thermalized spin-only quasiparticles of $\mathrm{Nb}$. Our experiment does not provide the mechanism for this process. The equilibration mechanism may be diffusion of the "hot" phonons of $\mathrm{Nb}$ across the barrier, inelastic scattering with phonons or electron-electron interactions. ${ }^{20}$ All these processes are favored by the low heat capacity of the Al.

We injected tunneling particles from a ferromagnet into a superconductor. The quasiparticles thermalize quickly to near the gap edge where the incoming charge goes into the condensate. This charge is drained by a supercurrent leaving a distribution of zero charge, spin-only (at the gap edge) quasiparticles. Because they carry no charge, these have a reduced spin-orbit scattering and therefore a long spinrelaxation time, which increases their recombination time so that they thermalize to a temperature different from the condensate. This consequence of the spin-charge separation is measured by probing the effective temperature of outgoing particles using a tunnel detector. No such rise in temperature is obtained when the same current is injected from a paramagnet rather than a ferromagnet.

The authors thank S. Caprara, M. Grilli, and P. Monod for stimulating discussions. B.L. gratefully acknowledges C.M. Varma for numerous discussions and fruitful suggestions, especially in interpreting the data in terms of spin-charge separation. The experiments were partly supported by the French Ministère de la Recherche through an "Action concertée incitative."
*Electronic address: Brigitte.Leridon@espci.fr

${ }^{1}$ S. Kivelson and D. Rokhsar, Phys. Rev. B 41, 11693 (1990).

${ }^{2}$ H. L. Zhao and S. Hershfield, Phys. Rev. B 52, 3632 (1995).

${ }^{3}$ M. Johnson, Appl. Phys. Lett. 65, 1460 (1994).

${ }^{4}$ R. Meservey and P. Tedrow, Phys. Rep. 238, 173 (1994).

${ }^{5}$ M. Tinkham, Phys. Rev. B 6, 1747 (1972).

${ }^{6}$ The general expression for the current derived in ${ }^{5}$ holds as well for polarized quasiparticles: $I(V)=(4 \pi e / \hbar)|T|^{2} D_{N}\left(E_{F}\right)$ $\times \int_{\Delta}^{\infty}\left\{\left[f_{k<}-f_{k>}\right]+D_{S}(E) \times[f(E-e V)-f(E+e V)]\right\} d E$, where $f_{k>}$ and $f_{k<}$ refer to the out-of-equilibrium occupation distributions for the quasielectron and quasihole branches, respectively, in the superconductor. The first term contribution does not carry any energy dependence, is expected to be small and is not able to account for the experimental observation.

${ }^{7}$ R. C. Dynes, V. Narayanamurti, and J. P. Garno, Phys. Rev. Lett. 41, 1509 (1978).

${ }^{8}$ W. H. Parker, Phys. Rev. B 12, 3667 (1975).
${ }^{9}$ D. M. Ginzberg, Phys. Rev. Lett. 8, 204 (1962).

${ }^{10}$ P. Hu, R. C. Dynes, and V. Narayanamurti, Phys. Rev. B 10, 2786 (1974).

${ }^{11}$ G. Sai-Halasz, C. C. Chi, A. Denenstein, and D. N. Langenberg, Phys. Rev. Lett. 33, 215 (1974).

${ }^{12}$ F. Jaworski and W. H. Parker, Phys. Rev. B 20, 945 (1979).

${ }^{13}$ M. Johnson, Phys. Rev. Lett. 67, 374 (1991).

${ }^{14}$ C. S. Owen and D. J. Scalapino, Phys. Rev. Lett. 28, 1559 (1972).

${ }^{15}$ J.-J. Chang and D. J. Scalapino, Phys. Rev. B 10, 4047 (1974).

${ }^{16}$ D. Vier and S. Schultz, Phys. Lett. 98A, 283 (1983).

${ }^{17}$ Y. Yafet, Phys. Lett. 98A, 287 (1983).

${ }^{18}$ S. B. Kaplan, C. C. Chi, D. N. Langenberg, J.-J. Chang, S. Jafarey, and D. J. Scalapino, Phys. Rev. B 14, 4854 (1976).

${ }^{19}$ V. Seminozhenko, Sov. Phys. Solid State 15, 1945 (1974).

${ }^{20}$ F. Pierre, H. Pothier, D. Estève, and M. Devoret, J. Low Temp. Phys. 118, 437 (2000). 